\title{
O papel mediador entre confiança e
}

\section{desempenho organizacional}

\author{
Marco Tulio Fundão Zanini \\ Fundação Getulio Vargas - Rio de Janeiro/RJ, Brasil \\ Carmen Pires Migueles \\ Fundação Getulio Vargas - Rio de Janeiro/RJ, Brasil
}

Recebido em 15/março/2012

Aprovado em 26/novembro/2012

Sistema de Avaliação: Double Blind Review Editor Cientifico: Nicolau Reinhard

DOI: $10.5700 /$ rausp 1130

\begin{abstract}
Neste artigo, busca-se contribuir para as discussões sobre a relação entre confiança e desempenho organizacional numa perspectiva econômica. Examinou-se o papel mediador entre confiança e desempenho organizacional a partir da análise da confiança como um elemento de coordenação informal dentro das organizações. Observou-se que a confiança interpessoal é um elemento central para a melhor compreensão dos contratos relacionais, como os contratos de trabalho, e que investimentos na criação e na manutenção de ambientes de trabalho baseados em confiança devem observar uma racionalidade econômica. Por meio de revisão na literatura para buscar compreender a necessidade e os benefícios de contextos sociais de alta e baixa confiança, concluiu-se que confiança é um elemento essencial para a execução de específicas tarefas organizacionais e observou-se sua relação mediadora com o desempenho.
\end{abstract}

Palavras-chave: confiança, coordenação informal, contrato relacional, desempenho organizacional.

\section{INTRODUÇÃO}

Confiança tem sido o foco de vários estudos da teoria organizacional (Arrow 1969, 1974; Barber, 1983; Granovetter, 1985; Gambetta, 1988; Burt, \& Knez, 1995; Mayer, Davis, \& Schoorman, 1995; Lane, \& Bachmann, 1996, 1998; Dirks, \& Ferrin, 2001; Zanini, 2005, 2007, 2011). Nos últimos anos, a confiança tem continuado como um tema que desperta grande interesse entre estudiosos e pesquisadores das organizações (Cook, Hardin, \& Levi, 2005; Bachmann, \& Zaheer, 2006; Frankel, 2006; Kramer, \& Cook, 2006; Reina, \& Reina, 2006; Schoorman, Mayer, \& Davis, 2007; Cook, Levi, \& Hardin, 2009; Dirks, Lewicki, \& Zaheer, 2009). Como resultado desse interesse, várias contribuições têm surgido de outras disciplinas das ciências sociais, incluindo ciência política, antropologia,
Marco Tulio Fundão Zanini, Mestre em Gestão Empresarial pela Escola Brasileira de Administração Pública e de Empresas da Fundação Getulio Vargas e Doutor em Management pela Universidade Otto-von-Guericke (Magdeburg, Alemanha), é Professor na Escola Brasileira de Administração Pública e de Empresas da Fundação Getulio Vargas (CEP 22250-900 - Rio de Janeiro/RJ, Brasil).

E-mail: marco.zanini@fgv.br

Endereço:

Fundação Getulio Vargas

Escola Brasileira de Administração Pública e de Empresas Praia de Botafogo $190-5^{\circ}$ andar

22250-900 - Rio de Janeiro - SP

Carmen Pires Migueles, Consultora Especialista em Diagnósticos de Cultura Organizacional e Avaliação de Ativos Intangíveis. Doutora em Sociologia das Organizações e Mestre em Antropologia do Consumo pela Universidade de Sophia (Tóquio, Japão), é Professora na Escola Brasileira de Administração Pública e de Empresas da Fundação Getulio Vargas (CEP 22250-900 - Rio de Janeiro/RJ, Brasil), E-mail: carmen.migueles@fgv.br 
sociologia, psicologia e economia (Cook et al., 2009). No Brasil, a partir de 2005, pode-se observar uma grande quantidade de estudos empíricos e teóricos sobre o tema, na forma de artigos e dissertações (Lazzarini, Madalozzo, Artes, \& Siqueira, 2005; Zanini, 2005; Cunha, \& Melo, 2006; Zanini, 2007, 2011; Zanini, \& Almeida, 2009; Andrade, Rezende, Salvato, \& Bernardes, 2011; Iwai, 2011; Frederico, 2012).

Entendendo que as organizações consistem basicamente em estruturas burocráticas e hierárquicas, a confiança torna-se um mecanismo informal para a coordenação e o controle da rotina organizacional (Ripperger, 1998; Wolff, 2000; Zanini, 2007, 2011). A existência de confiança nas relações burocráticas expande consideravelmente o potencial de produzir relações cooperativas e eficiência gerencial, por meio do aumento do controle gerencial gerado por um mecanismo informal (Zanini, 2007). Segundo Ripperger (1998), a presença da confiança nas relações interpessoais dentro da organização permite a redução do controle hierárquico formal, enquanto expande as possibilidades do emprego do conhecimento com maior grau de autonomia, facilitando a eliminação de redundâncias e processos imperfeitos e permitindo a melhoria contínua pela redução dos custos de transação. Assim, a confiança tem sido abordada como um elemento de coordenação informal essencial para a gestão das organizações (Wolff, 1996; Zanini, 2011).

A relação entre a variável confiança e muitas das variáveis do desempenho organizacional tem sido comprovada em diversos estudos. A existência de confiança entre os membros de uma empresa pode contribuir de forma significativa para o aumento da eficiência das diversas tarefas organizacionais (Dirks, \& Ferrin, 2001, 2002). Sua relevância tem sido observada principalmente nas tarefas mais específicas e complexas, que envolvem maior risco e incerteza (Luhmann, 1980, 2000; Ouchi, 1980; Coleman, 1990; Adler, 2001; Dirks, \& Ferrin, 2001). O papel informal da confiança na coordenação e no controle de diversas tarefas da organização tem sido observado, por exemplo, quando facilita o processo de transferência do conhecimento (Roberts, 2000; Rolland, \& Chauvel, 2000; Mesquita, \& Lazzarini, 2008; Jensen, \& Webster, 2009), melhora a eficiência e a produtividade organizacional (Akerlof, 1970; Arrow, 1974; Ouchi, 1980; Ring, \& Van De Ven, 1992; Bradach, \& Eccles, 1998; Lane, \& Bachmann, 1998; Sako, 1998) e diminuiu os custos de transação (Kreps, 1990; Chiles, \& McMackin, 1996; Butter, \& Mosch, 2003; Argyres, \& Mayer, 2007; Andrade et al., 2011). Segundo estudos empíricos realizados sobre o tema, quando existe relação de confiança entre as pessoas, cresce a probabilidade de trocas e compartilhamento de informações, reduzem-se os conflitos e aumentam a satisfação e a motivação (Dirks, \& Ferrin, 2001). Igualmente, diminuem os custos relacionados à aplicação excessiva de instrumentos burocráticos de segurança - como monitoração, regras e procedimentos formais. Nesse sentido, confiança pode agir ainda como um elemento facilitador em disputas para tomada de decisão, permitir maior flexibilidade de gerenciamento e diminuir a necessidade de mecanismos de controle (Xavier Molina-Morales, Teresa Martínez-Fernández, \& Torló, 2011; Horta, Demo, \& Roure, 2012). Como afirma Luhmann (1980), as relações de confiança geradas por normas sociais podem aumentar a eficiência das interações, operando como um mecanismo de redução de incertezas.

Muitos dos trabalhos acima relacionados ao tema apresentam a confiança como uma variável influenciando ou sendo influenciada por uma ou mais variáveis do desempenho organizacional. Falta, no entanto, uma perspectiva que possa ajudar a aprofundar mais o entendimento e a discussão sobre a relação entre confiança interpessoal e desempenho organizacional. Nesse sentido, no presente artigo, propõe-se a avançar nesta discussão: Como se pode melhor compreender a relação entre confiança interpessoal e desempenho organizacional? Essa é a pergunta investigativa que pauta a reflexão teórica, cuja relevância jaz no resgate e avanço da discussão sobre esse construto, conforme sugerido por Zanini (2011). Utilizando como metodologia de pesquisa a análise bibliográfica, buscou-se avaliar, na perspectiva econômica, a relação entre essas variáveis a partir da análise do papel da confiança na coordenação informal dos contratos relacionais ${ }^{(1)}$.

Para melhor compreender esse mecanismo social e suas consequências dentro das organizações, apresenta-se este artigo em seis seções. Na primeira, apresenta-se a introdução; na segunda seção, faz-se uma introdução sobre a confiança na perspectiva econômica; na terceira, define-se e ilustra-se o elemento confiança como um mecanismo de coordenação informal dentro das organizações. Na quarta seção, apresentam-se uma definição e a análise dos contratos relacionais e a confiança como seu elemento central. Na quinta seção, apresenta-se a relação entre confiança e desempenho organizacional. Finalmente, na ultima seção deste artigo, apresentam-se as conclusões.

\section{CONFIANÇA NA PERSPECTIVA ECONÔMICA}

Para melhor compreender a relação entre confiança e desempenho organizacional, adotou-se uma abordagem econômica. Segundo Williamson $(1985,1996)$, comparada com outras ciências sociais, a abordagem econômica para as organizações é mais "calculativa", podendo auxiliar na melhor compreensão dessa relação. Gibbons (2000) observa que o conceito "calculativo" da confiança baseado na Teoria da Decisão Racional torna-se útil para os estudos econômicos sobre o tema, apesar de o autor reconhecer igualmente que há mais sobre confiança do que um conceito meramente "calculativo". No entanto, afirma que tal conceito é importante para o estudo da confiança na atividade econômica (Gibbons, 2000, p. 20). Dessa forma, Gibbons (2000) observa que a perspectiva econômica busca compreender e abordar a confiança em termos econômicos, não rejeitando, porém, uma melhor compreensão social e relacional do objeto, igualmente importante para a economia. $\mathrm{O}$ foco em motivos meramente econômicos torna-se importante para 
construir um entendimento mais completo sobre os elementos da confiança nas questões econômicas, permitindo que pesquisadores, economistas e gestores possam compreender melhor, por exemplo, os efeitos da confiança nas hierarquias, como um mecanismo informal, como é o objetivo neste trabalho.

Ripperger (1998), sob a perspectiva econômica, entende que confiança é relacional e compreende um estado psicológico que reconhece bases cognitiva e afetivo-emocional como aspectos motivacionais para a decisão das pessoas em se engajarem em ações cooperativas. A autora observa que, mesmo quando se desenvolve sob forte base emocional, a confiança consiste em reações desenvolvidas sobre uma compreensão cognitiva. Uma vez que são desenvolvidas como reações de estruturas cognitivas, as emoções possuem um caráter compreensível. Assim, na perspectiva econômica, a compreensão cognitiva da confiança torna-se relevante. Como Hardin (1998, p. 11) afirma, "confiança é fundamentalmente cognitiva. Confiar ou desconfiar dos outros é ter alguma pressuposição de conhecimento sobre eles". Outra propriedade fundamental da confiança, largamente reconhecida por estudiosos de diversas áreas, é a ideia do risco comportamental (Luhmann, 1980; Wolff, 2000; Dirks, \& Ferrin, 2001; Zanini, 2007; Gillespie, \& Dietz, 2009), o que, na perspectiva econômica, pode ser traduzido em custo (Wolff, 2000, p. 1). Quanto mais existir o perigo do comportamento de risco e o oportunismo, mais a confiança se torna uma valiosa alternativa para a coordenação das hierarquias em sistemas econômicos.

$\mathrm{Na}$ perspectiva econômica, compreende-se a confiança como um mecanismo complementar, e não substituto, na relação com outros mecanismos formais de gestão (Luhmann, 1980; Ouchi, 1980; Van De Ven, \& Walker, 1984; Zucher, 1986; Aulakh, Kotabe, \& Sahay, 1996; Bradach, \& Eccles, 1998; Zanini, \& Almeida, 2009, Zanini, 2011). Em outras palavras, confiança (como um mecanismo informal) não é compreendida como uma opção equivalente à adoção de mecanismos formais de gestão, mas como um mecanismo complementar que deverá coexistir com eles. Nesse sentido, Van de Ven e Walker (1984) e Aulakh et al. (1996) concluem, em seus estudos empíricos, que o uso do controle formal tem consequências negativas nas relações de confiança entre agentes de interação. Os autores afirmam que a monitoração é compreendida como falta de confiança e, consequentemente, tem efeitos negativos para as relações entre as pessoas. Nesse sentido, Luhmann (1980) observa que o uso de sanções legais é geralmente percebido como incompatível com as relações de confiança. O autor observa que normas legais, quando empregadas, não substituem a função social da confiança; ao contrário, acabam por dirigir a expectativa dos atores sociais para formas de comportamentos menos cooperativas. Assim, Ouchi (1980) argumenta, observando a presença da confiança e do controle formal em diferentes níveis, que a análise dessa relação deverá definir formas mais eficientes de governança das organizações. Geralmente, a perspectiva econômica permite que se observem duas formas possíveis para analisar a eficiência da confiança e a consequente redução dos custos de transação. A primeira é compreender a confiança como um elemento que inibe o oportunismo e promove relações mutuamente benéficas, por motivar as pessoas a cooperarem umas com as outras sem a presença de mecanismos de controle e monitoração. Nesse caso, o mesmo resultado poderá ser alcançado de forma mais eficiente, a menores custos. A segunda é entender a confiança como um elemento que pode promover vantagem competitiva para se alcançarem resultados superiores, quando a confiança passa a tornar-se um elemento fundamental para se alcançarem os objetivos corporativos. Nesse caso, o resultado poderá ser maior. Assim, a decisão gerencial pela adoção de um estilo de gestão que favoreça as relações de confiança deverá observar a melhoria da eficiência organizacional. A decisão por adotar tal estilo de gestão irá solicitar investimentos na construção e manutenção de um contexto organizacional que favoreça a cooperação espontânea entre agentes, baseado em regras que sejam percebidas como mutuamente benéficas (Zanini, 2011).

\section{CONFIANÇA COMO MECANISMO DE COORDENAÇÃO INFORMAL}

Hardin (1998) afirma que relacionamentos de confiança são constituídos por:

- características pessoais de quem realiza um investimento de confiança - aqui se denomina $\mathbf{A}$ o indivíduo que confia;

- características pessoais de quem recebe o investimento de confiança - aqui se denomina $\mathbf{B}$ o indivíduo que recebe o investimento de confiança;

- específico contexto transacional em que ocorre determinada relação de confiança - denominado $\mathbf{X}$.

De acordo com Hardin (2002), a confiança consiste em um "interesse encapsulado", no sentido de que as expectativas do indivíduo que confia (A) estão baseadas na percepção que ele possui das reais motivações do indivíduo que recebe o investimento de confiança (B). Assim, mesmo que esses indivíduos possuam divergência na maioria de seus incentivos e interesses, a existência de algum ponto comum de interesse poderá estimular A a realizar um investimento de confiança em B. Além disso, Hardin (1998) reconhece a importância do contexto social. Segundo ele, dependendo do contexto específico $\mathbf{X}$, pessoas possuem mais ou menos incentivos para realizar investimentos de confiança. É importante observar que uma relação de confiança pode estabelecer-se sobre bases diferentes, de formas diferentes e em níveis diferentes: pode-se confiar nas pessoas de uma forma constante, para várias questões, ou confiar uma única vez, somente em relação a uma questão específica.

$\mathrm{Na}$ mesma linha de Hardin e de maneira complementar, Ripperger (1998) apresenta um trabalho que vai além da pura Teoria da Decisão Racional para construir um melhor entendi- 
mento sobre as relações de confiança, utilizando alguns pressupostos na forma de estados psicológicos. Ripperger (1998) contribui para um melhor entendimento sobre os investimentos de confiança em ações cooperativas, assumindo duas condições que precisam ser satisfeitas para haver uma relação de confiança: primeiro, a coexistência de uma expectativa de confiança e, num segundo momento, uma ação baseada em confiança. Para a autora, a existência desses dois componentes centrais caracteriza uma relação de confiança. Segundo Ripperger (1998), uma expectativa de confiança é definida como a expectativa de quem confia (A) em outra pessoa (B), acreditando que ela estará de fato motivada a não agir de forma oportunista. Assim, uma ação baseada em confiança é um investimento voluntário da pessoa que confia (A), na forma de ação concreta, sob situação de risco comportamental, sem recorrer a nenhum mecanismo explícito de segurança ou controle.

Ainda, conforme essa análise, confiança não pode ser entendida somente como uma predisposição ou expectativa, mas deve concretizar-se necessariamente em uma ação que envolve risco comportamental. Tal expectativa de confiança manifesta-se por meio de um estado subjetivo inicial, ou uma reação emocional, traduzido em uma condição cognitiva racional. Dessa forma, um indivíduo consegue avaliar e calcular sua predisposição em engajar-se numa determinada situação que envolva risco relativo ao comportamento de outro. Ripperger (1998) define confiança como aceitação voluntária e antecipada de um investimento de risco, pela abdicação de mecanismos contratuais explícitos de segurança e de controle, na expectativa de que a outra parte não agirá de forma oportunista. De acordo com Ripperger (1998), a expectativa de confiança, que é o pressuposto para uma ação baseada em confiança, baseia-se na percepção subjetiva do indivíduo que confia (A) das reais motivações (autointeresses percebidos) do indivíduo que recebe o investimento de confiança (B), em corresponder a seu investimento. Assim, complementando Hardin (2002, p. 3), o indivíduo que recebe o investimento de confiança (B) "encapsula" os interesses do indivíduo que confia (A) de forma a agir de maneira confiável.

De acordo com Ripperger (1998), a expectativa de confiança, na forma de um estado psicológico é constituída por dois elementos de forma dualística: um aspecto emocional e um aspecto cognitivo. Esses dois componentes coexistem e podem prevalecer um sobre o outro, de acordo com as pessoas com quem se interage e as situações específicas. Por vezes, uma expectativa de confiança está baseada mais em aspectos emocionais. Outras vezes, essa expectativa estará baseada mais em aspectos cognitivos. Dessa forma, agentes de interação poderão estar motivados de forma diferente, sobre bases mais emocionais ou mais cognitivas, de acordo com determinada pessoa ou grupo de pessoas, situações, contextos ou circunstâncias.

Ripperger (1998) comenta que a base cognitiva traz às expectativas do indivíduo que confia (A) a capacidade de calcular o risco relativo em determinada situação. Por outro lado, a base emocional traz aspectos afetivos e emocionais às expectativas do indivíduo que confia (A). Segundo a autora, o aspecto emocional de uma expectativa de confiança não pode ser rejeitado na análise das motivações para a realização de um investimento de confiança porque traz em si um aspecto fundamental e inerente a uma relação de confiança: a crença. Essa crença é necessária para superar a informação imperfeita causada pelas incertezas de uma penetração intelectual limitada de $\mathbf{A}$, o indivíduo que confia, sobre as reais motivações de $\mathbf{B}, \mathrm{o}$ indivíduo que recebe o investimento de confiança. No entanto, Ripperger observa que os elementos emocionais são reações desenvolvidas sobre uma compreensão cognitiva. Sentimentos pessoais construídos sob reações emocionais são desenvolvidos sobre uma compreensão cognitiva relativa às situações específicas. Como se desenvolvem como reações sobre estruturas cognitivas em situações específicas, emoções possuem um caráter compreensivo. Assim, embora cada expectativa de confiança traga em si aspectos cognitivos e emocionais, os aspectos cognitivos são preponderantes na análise das motivações e reações do indivíduo que recebe o investimento de confiança (B). Consequentemente, uma vez que uma relação de confiança é atribuída basicamente às motivações de $\mathbf{B}$ (acessado de forma imperfeita pela percepção de $\mathbf{A}$ ), cada evento de confiança deve primeiramente ser reconhecido pelas reais motivações de $\mathbf{B}$, mesmo que sob fortes circunstâncias emocionais. A Figura 1 ilustra a construção da expectativa de confiança baseada na percepção subjetiva de $\mathbf{A}$, sobre as motivações que B possui em honrar ou desonrar o investimento de confiança. As estruturas cognitivas e emocionais de A são influenciadas: pelas formas de percepção e características objetivas de determinada situação; pela informação adquirida de interações com B no passado (em forma de reputação); pelas informações sobre a específica situação no presente. Nesse sentido, observa-se um filtro que separa a percepção de $\mathbf{A}$ sobre as motivações de $\mathbf{B}, \mathrm{e}$ as reais motivações de $\mathbf{B}$. Com isso, pode-se reconhecer aqui o conceito de informação assimétrica nas relações de confiança. Assim, pode-se compreender que se realiza uma avaliação das outras pessoas com quem se interage com base na informação limitada que se tem sobre suas reais motivações ("interesses encapsulados") em agirem de forma a honrar o investimento de confiança.

A expectativa de confiança existe potencialmente, mesmo antes de se realizar uma ação concreta baseada nessa expectativa, na forma de certo grau de confiabilidade. Por essa razão, pode-se entender que A possui uma expectativa no comportamento de $\mathbf{B}$, independentemente da realização de uma ação concreta baseada em confiança. Dessa forma, pode-se entender que A possui certa predisposição de engajar-se voluntariamente em uma específica situação de risco com $\mathbf{B}$, independentemente das chances de interação entre ambos. Essa expectativa de confiança pode ser entendida como um elemento de motivação informal para uma possível ação concreta baseada em confiança. 


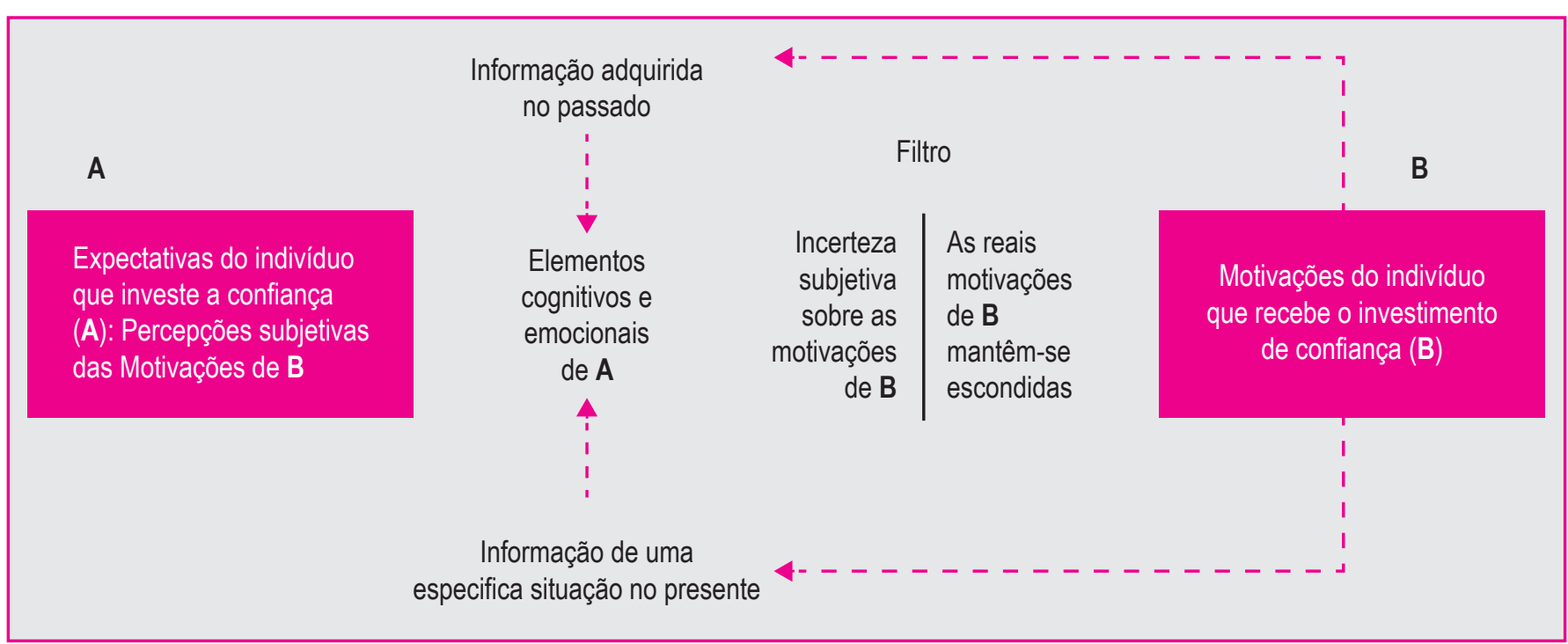

Fonte: Ripperger (1998, p. 95).

Figura 1: A Expectativa da Confiança

\section{CONTRATOS RELACIONAIS BASEADOS EM CONFIANÇA}

Tendo analisado o mecanismo da confiança entre agentes de interação na seção anterior, passa-se aqui a melhor compreender sua função nos contratos relacionais ${ }^{(2)}$. Confiança é reconhecida como um elemento central para uma melhor avaliação dos contratos relacionais (Kreps, 1990; Wolff, 1996; Casson, 1997; Gibbons, 2000, 2001; Reina, \& Reina, 2006). Contratos de trabalho são exemplos de contratos relacionais baseados em confiança interpessoal. Mesmo que necessitem ser firmados por meio de contratos explícitos - na configuração de um documento formal - por razões legais, esses contratos tornam-se contratos relacionais entre agentes de interação ao longo do tempo. A confiança que poderá ser construída como fruto dessa interação funciona como um mecanismo informal de controle e coordenação para diversas tarefas organizacionais. Em vez de antecipar todas as contingências futuras, os contratos relacionais antecipam uma série de incertezas, na forma de transações entre parceiros de interatividade, com base nas experiências passadas entre esses agentes. Por isso esses contratos são caracterizados como incompletos por definição. Os problemas contratuais que porventura possam surgir numa relação de trabalho poderão ser reduzidos de forma significativa com a presença da confiança.

Contratos relacionais são contratos incompletos, usados para explicar a cooperação num mundo de eventos futuros incertos (Furubotn, \& Richter, 2001). Por definição, contratos relacionais referem-se a relações contratuais de longo prazo, de forma que qualquer nova informação que se faça disponível, exogenamente ou endogenamente ao sistema, poderá gerar novas opções para os parceiros de interação, promovendo novos comportamentos (Wolff, 1996). Dessa forma, pode ser vantajoso para os parceiros de interação incorporar essa nova informação dentro do relacionamento, buscando assim um equilíbrio sequencial no tempo.

A moderna Teoria Contratual Econômica explica a transformação da ação individual na ação coletiva por meio do conceito da constituição corporativa (Wolff, 1996). Um dos principais tópicos da teoria é assegurar a eficiência das transações econômicas por meio da modelagem de mecanismos contratuais eficientes que incorporem incentivos e restrições. A teoria propõe que todo sistema de trocas ou interações humanas (ou seja, qualquer processo de produção ou previsão de serviços) pode ser descrito como uma relação contratual formal ou informal, que organiza as trocas de recursos ou serviços mediante outros benefícios. Diferentemente dos contratos bilaterais, a constituição corporativa consiste em contratos multilaterais. Wolff (1996) observa que a estipulação da constituição corporativa ocorre por contratos formais, que podem ser reforçados legalmente por terceiros, como os contratos de trabalho ou convenções corporativas escritas, ou por contratos informais, considerando a cultura corporativa de uma organização. A autora afirma que, apesar de não estarem escritos formalmente, esses aspectos informais da constituição corporativa formam uma parte essencial do conjunto de regras de uma organização. Assim, por definição, contratos relacionais são aqueles que não podem ser monitorados ou controlados por terceiros. Portanto, rearranjos contratuais são necessários ao longo do tempo de forma a assegurar um processo de cooperação contínua entre todos os parceiros de interação. De acordo com o contexto específico, a confiança pode ser altamente desejável e eficiente para lidar com as incertezas comportamentais causadas pelo surgimento de nova informação nas relações entre parceiros 
de interação. Quanto maior a frequência do surgimento de nova informação dentro do sistema, maiores serão as chances do aumento da incerteza comportamental, e mais necessário será o uso de contratos relacionais baseados em confiança, de forma a lidar com essa incerteza. Assim, a relevância e a eficiência da confiança, atuando como um mecanismo informal de governança, estão primariamente relacionadas ao grau de incerteza endógena e exógena, na forma da ausência ou confiabilidade da informação, ou na frequência do surgimento de nova informação dentro do sistema com que se possa alterar ou modificar o comportamento dos agentes. Além disso, mecanismos sociais como confiança são particularmente importantes quando o término de um relacionamento pode representar um custo muito alto (particularmente nos relacionamentos em que existe uma relação específica construída ao longo do tempo). Nesse sentido, os contratos de trabalho podem ser compreendidos como exemplos de contratos relacionais baseados em relacionamentos específicos. Esses podem combinar as características de contratos formais e informais, na forma de reputações construídas dentro de relacionamentos idiossincráticos.

Contratos de trabalho estão baseados em mecanismos que determinam seu cumprimento (self-enforcing) de forma a assegurar a eficiência das relações, exatamente pela dificuldade de monitoração e avaliação (Keefer, \& Knack, 2005). Podem ser mais bem compreendidos no modelo de reputações do comportamento racional, confrontado por contingências imprevisíveis (Kreps, 1990; Wolff, 1996). Nesse caso, a confiança gerada nos relacionamentos interpessoais funciona como um mecanismo de autocontrole, presente em determinado grau em todas as relações de trabalho. Há algum tempo Macaulay (1963) já afirmava que os homens de negócio buscam manter seu comprometimento baseado em sua reputação e amizades pessoais. A lógica reside no fato de que as partes concordam antecipadamente em cooperar no longo prazo porque ambas querem manter suas reputações para as futuras transações. Assim, numa abordagem econômica, a premissa central dos contratos relacionais é que eles são contratos baseados em confiança, que permitem a alocação ou absorção de riscos no compartilhamento de recursos. Nesse sentido, é possível observar nos contratos relacionais a coexistência de propriedades formais e informais, de forma a assegurar o comprometimento das partes para a geração de benefícios mútuos (Kreps, 1990; Wolff, 1996; Casson, 1997; Gibbons, 2000). Cada parte motivada a manter sua credibilidade e reputação com os outros agirá de forma cooperativa. Como Keefer e Knack (2005) observam, quando as partes confiam inerentemente umas nas outras, torna-se mais fácil as transações ocorrerem. A condição básica para isso é a manutenção das expectativas de continuidade das relações de vantagem mútua no longo prazo. Assim, a confiança torna-se elemento essencial em contratos relacionais, pois, como argumenta Arrow (1969), em sua ausência torna-se extremamente custoso buscar garantias e sanções alternativas, que possam inibir as oportunidades de cooperação mútua.
A ausência da confiança em contratos de trabalho pode significar a ausência de cooperação espontânea e representar o uso ineficiente de recursos humanos. Quando soluções judiciais são substituídas pela ordenação privada nas relações contratuais, a confiabilidade das promessas passa a solicitar comprometimentos confiáveis (credibilidade) que funcionam como o "cimento" dos contratos relacionais entre os indivíduos (Furubotn, \& Richter, 2001, p. 276). Comprometimentos confiáveis envolvem atos de reciprocidade que resguardam uma relação bilateral quando esses assumem a forma de investimentos irreversíveis (pela heterogeneidade, sem a possibilidade de utilizações múltiplas) e investimentos específicos (Williamson, 1985, 1996). Como se observou, relações de confiança mútua podem ser usadas como um dispositivo de segurança para construir comprometimentos confiáveis, na forma de reputações, por meio de investimentos específicos ao longo do tempo, quando indivíduos começam a usar estratégias de reciprocidade (Kreps, 1990; Wolff, 1996; Furubotn, \& Richter, 2001). Em Kreps (1990), os efeitos de reputações são construídos sobre normas sociais e princípios de justiça e benefícios mútuos que, por sua vez, devem prover aos hierarquicamente inferiores uma ideia sobre como a organização irá reagir em determinadas circunstâncias quando elas surgirem. Por fim, Williamson (1985) afirma que esses princípios devem dirigir comportamentos e expectativas dentro da empresa promovendo um condicionamento social relativo à segurança do emprego e à proteção contra a exploração.

Confiança está relacionada a interações repetidas e investimentos irreversíveis. Se suportarem a confiança investida, essas experiências tornam-se mecanismos que determinam o cumprimento do contrato, atenuando as chances de oportunismo entre as partes. Um sistema de reputações é construído sobre as relações de confiança ao longo do tempo definindo padrões de comportamento baseados na reciprocidade. Baseado nos estudos do comportamento coletivo em dilemas sociais usando Jogos Repetidos, Ostrom (2003) apresenta um esquema (Figura 2) que contém as variáveis centrais para a manutenção das relações de confiança.

O modelo apresentado por Ostrom (2003) considera variáveis físicas, culturais e institucionais como críticas para a formação do contexto em que as interações humanas ocorrem, podendo promover ou inibir investimentos de confiança. Assim, a distância física entre agentes de interação, modelos comportamentais que incentivam ou inibem a cooperação, ou a natureza dos sistemas produtivos (que podem necessitar mais ou menos da interatividade entre os indivíduos para a produção de determinado valor) constituem elementos fundamentais para a análise das relações de confiança. Essas, por sua vez, são sustentadas por regras informais de reciprocidade e pela reputação entre os agentes. Reciprocidade e reputação irão influenciar diretamente a percepção de risco, inibindo mais ou menos as relações de confiança, que influenciarão os níveis de cooperação e a capacidade de determinado sistema social produzir ganhos individuais e coletivos. 


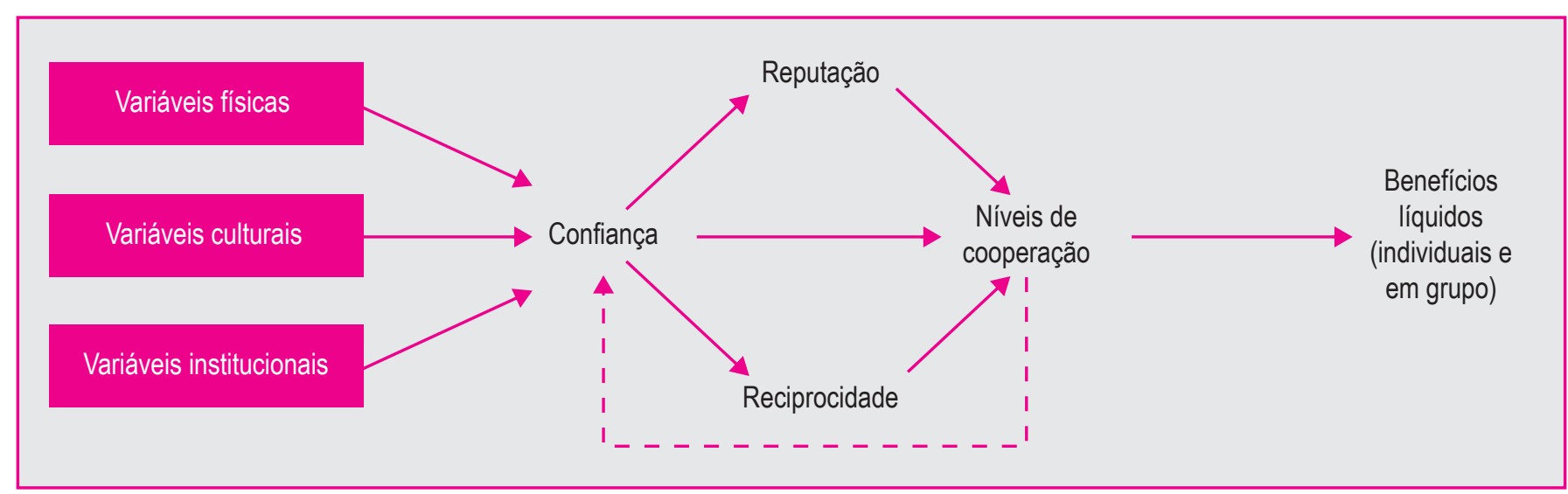

Fonte: Ostrom (2003, p. 51).

Figura 2: Relações de Feedback em Dilemas Sociais Repetidos

As pessoas em geral usarão de suas experiências do passado como informação para agir no presente. Quanto mais as pessoas receberam benefícios no passado como fruto de interações com outros parceiros, maiores serão suas inclinações para usar as estratégias de reciprocidade no presente. Quanto mais frequentemente as pessoas agem com base em reciprocidade, menos inclinações terão para desertar. Isso significa que, em geral, os agentes de interação que agem sob uma lógica de reciprocidade tenderão a confiar mais no presente que os outros irão agir igualmente de forma recíproca, com base em suas próprias normas pessoais afirmadas sobre as experiências do passado. No entanto, isso será afetado pela informação que os indivíduos poderão ter sobre a reputação de seus parceiros de interação no presente, e com isso poderão avaliar o risco de investir confiança em determinada situação específica.

Assim, confiança pode ser vista como uma forma racional de cooperação sob risco comportamental. Nesse sentido, empresas podem ser entendidas como redes de contratos relacionais que envolvem seus membros corporativos. De acordo com Furubotn e Richter (2001), empresas são constituições sociais que envolvem, além de suas regras, investimentos no cultivo de relacionamentos entre indivíduos. Os autores afirmam que o fortalecimento da cultura organizacional, como um mecanismo de compartilhamento de informação e redução do risco comportamental baseado em confiança mútua, representa um importante objetivo para os investimentos realizados. Assim, os níveis de confiança dentro das empresas podem ser entendidos como indicadores da eficiência da gestão dos contratos relacionais. Uma das funções das redes de contratos relacionais baseadas em confiança mútua é a capacidade de subsistir às incertezas futuras do mercado. Esse raciocínio indica que a presença de confiança torna-se relevante, por exemplo, em processos de mudança em geral, tais como processos de rápido crescimento ou downsizing, fusões e aquisições, e processos sucessórios, pois esses movimentos tendem a transferir incer- teza às relações sociais internas à empresa (Beckert, Metzner, \& Roehl, 1998). Nesses casos, a confiança existente poderá assegurar o cumprimento das expectativas entre as partes.

\section{RELAÇÕES ENTRE CONFIANÇA E DESEMPENHO ORGANIZACIONAL}

Após compreender-se a relevância da confiança nos contratos relacionais, como os contratos de trabalho, analisar-se-á nesta seção como a presença da confiança pode contribuir para o desempenho nas organizações. Estudos empíricos frequentemente têm sugerido que confiança possui um efeito mais moderador com o desempenho organizacional, do que uma relação direta e positiva (Dirks, \& Ferrin, 2001; Aryee, Budhwar, \& Chen, 2002; Zanini, 2007; MacCurtain, Flood, Ramamoorty, West, \& Dawson, 2009). Por exemplo, confiança é observada por Aryee et al. (2002) como elemento mediador para a percepção coletiva de justiça distributiva e procedimental, satisfação do trabalho e intenções de turnover. Esses estudos têm relacionado os efeitos positivos de confiança, por exemplo, nas relações entre líderes e liderados (Dirks, 2000, 2006; Dirks, \& Ferrin, 2002), no processo de transferência do conhecimento (Roberts, 2000; Rolland, \& Chauvel, 2000; MacCurtain et al., 2009), para fortalecer o comprometimento dos empregados (Brockner, Siegel, Tyler, \& Martin, 1997; Pillai, Schriesheim, \& Williams, 1999; Ugboro, 2003), e aumentar a eficiência e a produtividade organizacional (Ring, \& Van De Ven, 1992; Lane, \& Bachmann, 1998; Sako, 1998). Dirks e Ferrin (2001) apresentam uma meta-análise com uma série de estudos empíricos que confirmam os efeitos positivos das relações de confiança nas atitudes e comportamentos dos indivíduos dentro das organizações. De acordo com esses autores, espera-se que níveis superiores de confiança resultem em atitudes mais positivas, altos níveis de comportamento cooperativo espontâneo $\mathrm{e}$, consequentemente, desempenho superior. 
No entanto, isso não significa que níveis superiores de confiança determinem necessariamente desempenho superior, ou mesmo que empresas que apresentem baixos índices de confiança irão necessariamente apresentar baixo desempenho organizacional. É importante que a análise da relação entre os níveis de confiança e as variáveis do desempenho organizacional leve em consideração o contexto ou ambiente institucional em que essas transações ocorrem para que mecanismos de governança alternativos possam ser considerados numa análise custo-benefício (Zanini, 2007). Como se observou, a adoção de um estilo de gestão baseado em confiança possui custos inerentes ao estabelecimento de um contexto organizacional em que sejam criados e mantidos alguns elementos antecedentes e fundamentais para a construção de relacionamentos baseados em confiança. Por exemplo, Langfred (2004) estudou a relação entre confiança, monitoração e autonomia individual em equipes autogeridas e conclui que excessivos níveis de confiança podem tornar-se prejudiciais para a eficiência das equipes. Langfred conclui que, sob alta autonomia individual e altos níveis de confiança, a confiança em equipes autogerenciadas torna-se ineficiente, pois as pessoas nessas equipes tornam-se relutantes em monitorar umas às outras.

Como se observou no início deste artigo, a noção de confiança traz em si uma noção de risco comportamental associado, o que na perspectiva econômica significa custos associados (Coleman, 1990; Wolff, 2000). Portanto, um ambiente de trabalho baseado em relações de confiança necessita de consecutivos investimentos na manutenção de uma relativa estabilidade das condições e perspectivas de continuidade dos contratos de trabalho. A percepção coletiva de justiça nos procedimentos, na mensuração e na distribuição dos resultados torna-se igualmente fator crítico para a gestão dos níveis de confiança. Whitener, Brodt, Korsgaard e Werner (1998) apresentam um estudo sobre alguns elementos diretamente relacionados à construção de ambientes que favoreçam relações de confiança. Esses elementos são: a qualidade da comunicação interna, a percepção de integridade, a consistência e a preocupação com os empregados, além da delegação e do compartilhamento da autoridade. Esse conjunto de variáveis oferece igualmente melhor avaliação desses ambientes e da gestão dos bens intangíveis. Porém, ainda que tal estilo de gestão baseado em confiança possa ser altamente desejado para o cumprimento de determinadas tarefas organizacionais, $\mathrm{o}$ contexto institucional em que a empresa se encontra deve ser observado (Zanini, 2007).

Assim, o desenvolvimento da confiança consome tempo e requer investimentos específicos para sua emergência e manutenção dentro de uma empresa. Para que uma empresa adote um estilo de gestão baseado em confiança, será necessária a manutenção de incentivos que suportem relacionamentos de confiança, tais como normas de comportamento, conduta e punição, transparência organizacional, clareza na comunicação corporativa. Dessa forma, investimentos específicos poderão assumir a forma de treinamentos específicos, políticas de seleção mais rígidas para a contratação de executivos e manutenção de baixos níveis de rotatividade de empregados, criação e reforço de códigos de ética e de conduta, e criação e manutenção de canais de comunicação confiáveis entre a alta gestão e os empregados.

O grande benefício de ambientes de trabalho com uma atmosfera de alta confiança é permitir que indivíduos realizem transações sem a necessidade de se precaverem contra eventuais comportamentos oportunistas dos outros, aceitando assim maiores riscos. Em atmosferas de baixa confiança pessoas serão mais receosas em realizar investimentos de confiança umas nas outras, porque percebem baixos níveis de motivação nos parceiros de interatividade em adotarem estratégias de reciprocidade e, portanto, maior a probabilidade de assumirem prejuízos pessoais. No entanto, deve-se observar que, em específicos contextos organizacionais, pode ser possível que o fomento à competição interna entre os membros da empresa seja mais eficiente e produza melhores resultados. Lazear (1998) observa que, quando a eficiência das unidades de negócios está relacionada a um tipo de estratégia que incentiva a competição interna, esse processo permite a seleção natural de indivíduos e poderá privilegiar os esforços individuais em detrimento dos esforços coletivos. Portanto, em alguns casos, é possível que o resultado da soma da ação isolada de cada agente, e não o resultado das interações entre os agentes, possa ser mais eficiente para alcançar determinados objetivos, ainda que sob um contexto de baixa confiança. Nesses casos, o sistema de remuneração irá privilegiar e premiar os esforços individuais e provavelmente um estilo de gestão que estimule a competição entre os agentes em detrimento do desenvolvimento das relações de confiança. Por outro lado, onde se estimula a competição interna, torna-se extremamente difícil a criação de laços de confiança entre os agentes, e passa-se a contar menos com os benefícios desse mecanismo social de gestão. Ouchi (1980) e Osterloh e Frey (2000) afirmam que, em determinadas situações, quando os resultados são relativamente fáceis de ser mensurados, quando as tarefas possuem baixa complexidade e não requerem criatividade, velocidade de aprendizado, entendimento conceitual, ou não necessitam da transferência crítica do conhecimento, a aplicação de mecanismos formais como incentivo poderá ser relativamente mais eficiente. Por outro lado, esse não parece ser o caso quando sistemas produtivos necessitam do compartilhamento de informação sensível de forma a contribuir para a execução de tarefas especializadas e de maior complexidade, e promover ganhos por meio da interatividade de grupo de trabalho (Ouchi, 1980; Adler, 2001; MacCurtain et al., 2009). Como afirmam alguns autores (Osterloh, \& Frey, 2000; Adler, 2001), quando as tarefas organizacionais estão baseadas na inovação, na criação e no desenvolvimento de produtos, os melhores resultados poderão ser alcançados por meio da intensa interação entre as pessoas.

Outro ponto importante para a escolha de um estilo de gestão baseado em confiança é quando as medidas de desempenho tornam-se por demais ambíguas. Ouchi (1980) comenta que, nesses casos, o uso de confiança torna-se fundamental para 
o alinhamento dos objetivos individuais e organizacionais. Quando os mecanismos burocráticos tradicionais, baseados em controle formal, podem falhar, porque se torna impossível avaliar externamente o valor adicionado por indivíduo, o uso de confiança é mais recomendável. Nesse sentido, Ouchi (1980) e Dasgupta (2000) observam que a possibilidade de monitorar as ações dos indivíduos é um fator crucial pela decisão entre mecanismos explícitos de controle ou confiança. À medida que as tarefas se tornam mais complexas, interdependes ou ambíguas, reduz-se a capacidade de monitoração e avaliação do desempenho individual. Nesses casos, os contratos passam a depender mais dos acordos informais entre os agentes e necessitam da confiança para que as expectativas sejam atendidas.

Alguns estudiosos observam que o intenso uso de mecanismos explícitos de controle, como monitoramento formal, possui grande probabilidade de aumentar as chances de oportunismo (Van De Ven, \& Walker, 1984; Aulakh et al., 1996). Em outras palavras, a ênfase demasiada em garantias explícitas pode prejudicar consideravelmente o desenvolvimento de relações de confiança, o que pode ser custoso porque o uso de confiança não requer despesas adicionais com garantias legais. Onde níveis de confiança se fazem presentes, a necessidade de monitoração explícita e controle, e seus custos associados, será reduzida. Além disso, o uso intensivo de mecanismos explícitos de controle envolve não somente investimentos em monitoração e controle formal, mas provavelmente uma perda considerável da motivação dos indivíduos para gerarem comprometimentos em direção ao atendimento dos objetivos organizacionais (Ripperger, 1998). Empiricamente, encontrar-se-ão sempre a combinação e a coexistência de mecanismos formais e informais agindo em níveis diferentes dentro dos ambientes de negócios de forma complementar (Ouchi, 1980; Bradach, \& Eccles, 1998; Zanini, 2007).

Como se observou, à medida que as tarefas organizacionais se tornam mais complexas, incertas, ambíguas ou interdependentes, aumenta a necessidade da confiança entre os agentes de interação (Ouchi, 1980). Nesse sentido, a eficiência na execução das tarefas organizacionais dessa natureza dependerá em grande parte da confiança existente entre os agentes de interação (por exemplo, na relação entre gestores e subordinados, parceiros, colegas de trabalho ou entre agentes em equipes multifuncionais). À medida que o bem a ser produzido solicita a participação e a contribuição de vários especialistas, torna-se mais difícil mensurar a contribuição individual de cada agente. Quando a avaliação individual se torna demasiadamente ambígua, ela dependerá da confiança que os agentes de interação possuem em seus avaliadores ou no sistema de avaliação. Assim como no caso de tarefas mais complexas, cujos resultados a serem obtidos possuem alto grau de imprevisibilidade. Por outro lado, quanto menos complexas, incertas, interdependentes e ambíguas forem as tarefas, ou seja, quanto mais fáceis de mensurar e mais previsíveis forem os resultados a serem alcançados, menor a necessidade da confiança. Esses casos sugerem que os contratos formais poderão ser utilizados relativamente com maior eficiência.

Finalmente, torna-se importante observar a relação entre as variáveis institucionais para melhor compreender-se a relação entre os níveis de confiança e as variáveis do desempenho organizacional. Em última análise, como afirma Hadfield (2005), o que define primordialmente essa relação de custo e eficiência são os diversos arranjos institucionais que se traduzem em incertezas ambientais. Zanini (2007) apresenta um estudo em que avalia os níveis de incertezas institucionais, os níveis de confiança dentro das empresas e outras variáveis organizacionais. Confiança foi mensurada em três dimensões: confiança no superior, confiança em um colega de trabalho e confiança na equipe de trabalho. $\mathrm{O}$ estudo observou que altos níveis de confiança podiam ser encontrados em indústrias mais tradicionais, como metalúrgica e mineração, que apresentavam demandas relativamente estáveis, manifestadas por contratos de longo prazo com seus clientes e baixos níveis de rotatividade de empregados. Essas empresas apresentaram relativamente menores níveis de incerteza ambiental. Nelas os empregados demonstravam igualmente altos níveis de comprometimento e satisfação. Caracteristicamente, os empregados possuíam relacionamentos de longo prazo com suas empresas. Em média eles passavam dez anos ou mais na mesma empresa. Apresentavam altos níveis de confiança em seus superiores, seus colegas e suas equipes de trabalho. Por outro lado, as novas empresas orientadas por tecnologia no setor da informação e comunicação, que apresentavam relativamente altos níveis de incerteza ambiental, tinham menores níveis de confiança, maior rotatividade de empregados e menor comprometimento dos empregados com a empresa (Figura 3).

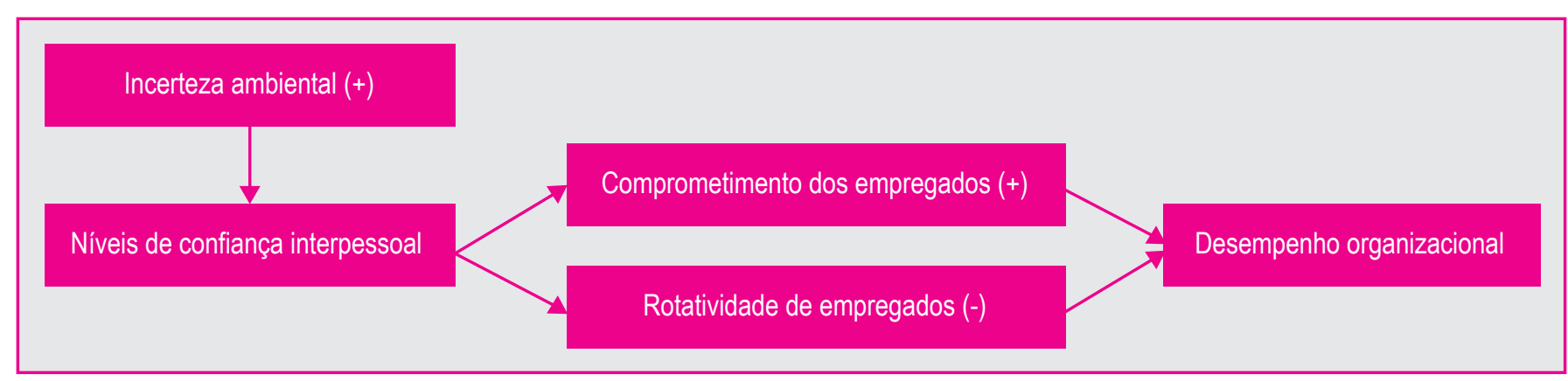

Figura 3: Incerteza Ambiental Influenciando Confiança e Fatores Organizacionais 
Esses resultados encontram-se em linha com os estudos anteriores de Van den Steen (2003), que observa que a excessiva rotatividade dos empregados enfraquece a cultura corporativa uma vez que os novos empregados não possuem a mesma informação dos mais antigos. Cotton e Tuttle (1986) realizaram uma meta-análise por meio de revisão na literatura e observaram que a rotatividade dos empregados (além do nível considerado "saudável") possui uma forte relação negativa com a satisfação no trabalho, satisfação com o trabalho em si, satisfação com a remuneração, com a liderança e com o comprometimento organizacional.

Nesse sentido, para avaliar como a confiança pode tornar-se um ativo intangível relevante e um fator distintivo no desempenho das organizações, propõe-se o modelo analítico a seguir (Figura 4). No modelo desenvolvido em estudos anteriores (Zanini, 2007, 2011), basicamente duas variáveis devem ser observadas: o nível de incerteza ambiental e a natureza das tarefas de organização. A Figura 4 ajuda a compreender melhor a relação entre a incerteza ambiental, os níveis de confiança e o desempenho organizacional.

O primeiro fator a ser observado é como o ambiente institucional se relaciona com o setor específico em que a empresa opera. Cada ambiente empresarial possui um determinado grau de incerteza. O grau de incerteza gerada pelo ambiente externo pode ser caracterizado principalmente por três fatores: a intensidade da concorrência (alta e baixa), que influencia e gera a necessidade da velocidade em apresentar respostas ao mercado; a estabilidade da demanda e da tecnologia (alta e baixa), que influencia a frequência da reorganização interna e a necessidade de adaptação, bem como gera novas demandas por tecnologias de produção e design de novos produtos; a

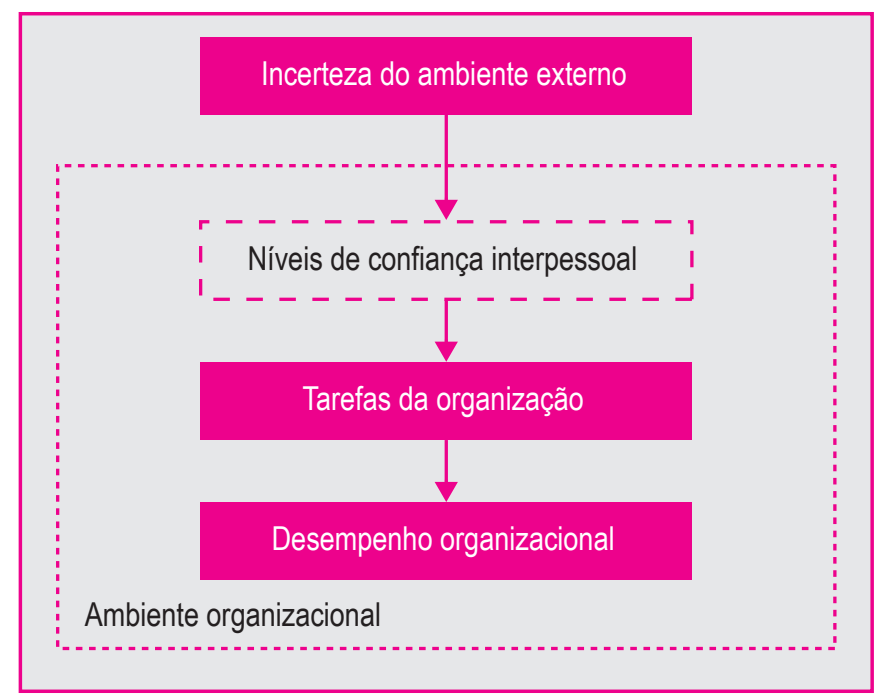

Fonte: Zanini (2011, p. 340). Figura 4: Incerteza Organizacional Influenciando a
Confiança e o Desempenho Organizacional capacidade de inovação que irá afetar diretamente a natureza dos contratos da empresa (por exemplo, se eles são de curto, médio ou contratos de longo prazo). Essas variáveis indicam o grau de incerteza institucional e influenciam a taxa de mudança e a necessidade de a empresa adaptar-se às novas demandas do mercado (Audretsch, \& Thurik, 2001). Elas também indicam o nível necessário de flexibilidade organizacional e a taxa de reposição e reorganização dos recursos da empresa, incluindo os recursos humanos. Consequentemente, a incerteza institucional influencia fortemente o comportamento dos indivíduos dentro da empresa, alterando suas expectativas futuras e impactando suas estratégias pessoais. Assim, a incerteza ambiental afeta consideravelmente o comportamento organizacional pela mudança das condições e expectativas das pessoas, podendo também mudar-lhes a disposição de confiarem umas nas outras. Portanto, a incerteza deve ser observada como um fator institucional restritivo no processo de construção de confiança (Zanini, 2011).

\section{CONCLUSÕES}

Apesar de, nos últimos anos, muitos estudos empíricos e teóricos terem avançado no tema sobre a relação entre a confiança interpessoal e as variáveis do desempenho organizacional, neste artigo adotou-se uma perspectiva econômica para aprofundar as discussões sobre o tema, de forma a melhor compreender essa relação a partir da análise do papel da confiança nos contratos relacionais. Dessa forma, utilizando a análise bibliográfica, procurou-se melhor compreender o papel da confiança dentro das organizações a partir da análise da confiança na coordenação informal dos contratos relacionais e, posteriormente, sua relação com as variáveis do desempenho organizacional. A análise ocorreu em três momentos distintos: na primeira seção, analisaram-se algumas propriedades da confiança de forma a melhor compreender seu funcionamento; em seguida analisou-se a confiança como elemento central para os contratos relacionais - como os contratos de trabalho que partem inicialmente de um dispositivo contratual legal e, posteriormente, desenvolvem-se numa perspectiva relacional ao longo do tempo. Identificou-se a confiança como elemento central para a eficiência desses contratos relacionais. Na última parte deste artigo, passou-se então a compreender a relevância da confiança no desempenho de tarefas específicas dentro das organizações e concluiu-se que a confiança pode ser mais bem compreendida como uma variável mediadora que contribui para a coordenação de específicas tarefas organizacionais, porém, não determinante para o desempenho organizacional. Nessa perspectiva, observou-se a relação eficiente entre a natureza específica das tarefas organizacionais (complexidade, incerteza, ambiguidade e interdependência) e a necessidade da confiança (ou controle formal) para a gestão dos contratos de trabalho. Observou-se que, para melhor compreender a relação entre confiança e desempenho organizacional, devem observar-se 
ainda as variáveis institucionais (físicas, culturais e institucionais) que podem se traduzir em incertezas do ambiente externo, com significativo impacto sobre o comportamento dos indivíduos nas organizações. Assim, buscou-se avançar nas discussões sobre o tema.

Este estudo possui limitações que representam, igualmente, possibilidades para o desenvolvimento de futuras pesquisas sobre o tema. Principalmente, faltam-lhe comprovações teóricas para o modelo analítico proposto. Também, não se aprofundou a análise da efetividade da inter-relação da confiança, como elemento informal dos contratos relacionais, com os elementos formais de coordenação. Também não se fez uma análise mais completa das variáveis institucionais que representam incertezas ambientais, o que poderia ajudar a compreender melhor outras perspectivas na análise da confiança como elemento de coordenação informal. Dessa forma, os resultados abrem espaço para novas pesquisas sobre o tema. Confiança é um elemento a ser mais bem compreendido e a compreensão de sua relação com o desempenho organizacional inaugura um vasto campo para futuras pesquisas.
(1) O que se chama aqui de mecanismos de coordenação informal refere-se aos aspectos não estruturais das organizações que Chester Barnard aborda como relevantes já em 1938, e que mais tarde são tratados como os aspectos políticos e simbólicos das organizações. Ver: Barnard (1938), Ramos (1981) e Williamson (1995).

(2) Gibbons (2001) observa que contratos relacionais por vezes são chamados por outros autores de self-enforcing, contratos informais ou contratos implícitos.

Audretsch, D. B., \& Thurik, A. R. (2001, January). What's new about the new economy? Sources of growth in the managed and entrepreneurial economies, working paper. Institute for Development Strategies (IDS), Indiana University Trustees.

Aulakh, P. S., Kotabe, M., \& Sahay, A. (1996). Trust and performance in cross-border marketing partnerships: a behavioural approach. Journal of International Business Studies, 27(4), 1005-1032. doi: 10.1057 /palgrave.jibs.8490161

Bachmann, R., \& Zaheer, A. (2006). Handbook of trust research. Northampton, MA: Edward Elgar.

Barber, B. (1983). The logic and limits of trust. New Brunswick, NJ: Rutgers University Press.

Barnard, C. (1938). The functions of the executive. Cambridge: Harvard University Press.

Beckert, J., Metzner, A., \& Roehl, H. (1998). Vertrauenserosion als organisatorische Gefahr und wie ihr zu begegnen ist. Organisationsentwicklung, 17, pp.56-66.

Bradach, J. L., \& Eccles, R. G. (1998). Price, authority and trust: from ideal types to plural forms, In G. Thompson et al. (Eds.), Markets, hierarchies \& networks - the coordination of social life (pp. 277-292). London: Sage.

Brockner, J. P., Siegel, P., Tyler, T., \& Martin, C. (1997). When trust matters: the moderating affect of outcome favorability. Administrative Science Quarterly, 42(3), 558-583. doi: $10.2307 / 2393738$ 
Burt, R., \& Knez, M. (1995). Kinds of third-party effects on trust. Journal of Rationality and Sociology, 7(3), 255-292. doi: $10.1177 / 1043463195007003003$

Butter, F. A. G., \& Mosch, R. H. J. (2006, October). Trade, trust and transaction costs. Department of Economics, Vrije Universiteit Amsterdam, and Tinbergen Institute, Discussion paper. Recuperado em 20 janeiro, 2012, de http://www. handelsland.nl/wp-content/uploads/2009/08/Tratrutra7.pdf

Casson, M. (1997). The economics of business culture game theory, transaction costs, and economic performance. Oxford: Clarendon Press.

Chiles, T. H., \& McMackin, J. F. (1996). Integrating variable risk preference, trust and transaction cost economics. Academy of Management Review, 21(1), 72-99. doi: 10.2307/258630

Coleman, J. (1990). Foundations of social theory. Boston: Belknap Press of Harvard University Press.

Cook, K. S., Hardin, R., \& Levi, M. (2005). Cooperation without trust. New York: Russell Sage Foundation.

Cook, K. S., Levi, M., \& Hardin, R. (2009). Whom can we trust: how groups, networks, and institutions make trust possible. New York: Russell Sage Foundation.

Cotton, J., \& Tuttle, J. (1986, January). Employee turnover: a meta-analysis and review with implications for research. The Academy of management Review, 11(1), 55-70. doi: 10.5465/AMR.1986.4282625

Cunha, C., \& Melo, M. (2006). A confiança nos relacionamentos interorganizacionais: o campo da biotecnologia em análise. RAE Eletrônica, 5(2). doi: 10.1590/S1676-56482006000200009

Dasgupta, P. (2000). Trust as a commodity. In D. Gambetta (Ed.), Trust: making and breaking cooperative relations (pp. 49-72, cap. 4). Oxford: University of Oxford.

Dirks, K. T. (2000). Trust in leadership and team performance: evidence from NCAA basketball. Journal of Applied Psychology, 85(6), 1004-1012. doi: 10.1037//0021-9010.85.6.1004

Dirks, K. T. (2006). Three fundamental questions regarding trust in leaders. In R. Bachmann \& A. Zaheer (Eds.), Handbook of trust research (pp. 15-28). Northampton, MA: Edward Elgar.

Dirks, K. T., \& Ferrin, D. L. (2001). The role of trust in organizational settings. Organization Science, 12(4), 450-467. doi: 10.1287/orsc.12.4.450.10640

Dirks, K. T., \& Ferrin, D. L. (2002). Trust in leadership: meta-analytic findings and implications for research and practice. Journal of Applied Psychology, 87(4), 611-628. doi: 10.1037//0021-9010.87.4.611

Dirks, K. D., Lewicki, R. J., \& Zaheer, A. (2009). Repairing relationships within and between organizations: building a conceptual foundation. Academy of Management Review, 34(1), 68-84.
Frankel, T. (2006). Trust and honesty: America's business culture at a crossroad. New York: Oxford University Press.

Frederico, J. M. B. (2012). A relação entre confiança, idade e escolaridade no ambiente organizacional. Dissertação de Mestrado Profissional, Ebape/Fundação Getulio Vargas, Rio de Janeiro, RJ, Brasil.

Furubotn, E. G., \& Richter, R. (2001). Institutions and economic theory - the contributions of the new institutional economics. Michigan: The University of Michigan Press.

Gambetta, D. (1988). Can we trust trust? In D. Gambetta (Ed.). Trust: making and breaking cooperative relationships (pp. 213-237). Cambridge, MA: Basil Blackwell.

Gibbons, R. (2000). Trust in social structures: Hobbes and Coase meet repeated games. In K. Cook (Ed.), Trust in society. New York: Russell Sage Foundation.

Gibbons, R. (2001, June). Relational contracts and the theory of the firm. Massachusetts: MIT Sloan School and NBER. Working Paper.

Gillespie, N., \& Dietz, G. (2009). Trust repair after an organization-level failure. Academy of Management Review, 34(1), 127-145. doi: 10.5465/AMR.2009.35713319

Granovetter, M. (1985). Economic action and social structure: the problem of embeddedness. American Journal of Sociology, 91(3), 481-510. doi: 10.1086/228311

Hadfield, G. K. (2005). The many legal institutions that support contractual commitments. In C. Ménard \& M. M. Shirley (Eds.), Handbook of new institutional economics (pp. 175-204), New York: Springer.

Hardin, R. (1998). Trust in government. In V. Braithwaite \& M. Levi (Eds.), Trust and governance (vol. 1, pp. 09-27, Series of Trust). New York: Russell Sage Foundation.

Hardin, R. (2002). Trust and trustworthiness. New York: Russell Sage Foundation.

Horta, P., Demo, G., \& Roure, P. (2012). Políticas de gestão de pessoas, confiança e bem-estar: estudo em uma multinacional. Revista de Administração Contemporânea, 16(4), 566-585.

Iwai, T. (2011). Bases de confiança e tipos de relacionamento entre agentes: um estudo sobre seus efeitos na reação cognitiva e comportamental dos agentes. Tese de Doutorado, EAESP/Fundação Getulio Vargas, São Paulo, SP, Brasil.

Jensen, P., \& Webster, E. (2009). Knowledge management: does capture impede creation? Industrial and Corporate Change, 18(4), 701-727. doi: 10.1093/icc/dtp025)

Keefer, P., \& Knack, S. (2005). Social capital, social norms and the new institutional economics. In C. Ménard \& M. M. Shirley (Eds.), Handbook of new institutional economics (pp. 701-726). New York: Springer. 
Kramer, R. M., \& Cook, K. S. (2006). Trust and distrust within organizations. New York: Russell Sage Foundation.

Kreps, D. M. (1990). Corporate culture and economic theory. In D. M. Kreps et al., Perspectives on positive political economy. Cambridge: Cambridge University Press.

Lane, C., \& Bachmann, R. (1996). The social constitution of trust: supplier relations in Britain and Germany. Organizations Studies, 17(3), 365-395. doi: $10.1177 / 017084069601700302$

Lane, C., \& Bachmann, R. (1998). Trust within and between organizations - conceptual issues and empirical applications. Oxford, UK: Oxford University Press.

Langfred, C. W. (2004, June). Too much of a good thing? Negative effects of high trust and individual autonomy in self-managing teams. Academy of Management Journal, 47(3), 385-399, doi: 10.2307/20159588

Lazear, E. P. (1998). Personnel economics for managers. New York: Wiley.

Lazzarini, S., Madalozzo, R., Artes, R., \& Siqueira, J. (2005). Measuring trust: an experiment in Brazil. Economia Aplicada, 9(2), 153-169.

Luhmann, N. (1980). Trust: a mechanism for the reduction of social complexity. In N. Luhmann, Trust and power. New York: Wiley.

Luhmann, N. (2000). Familiarity, confidence, trust: problems and alternatives. In D. Gambetta (Ed.), Trust: making and breaking cooperative relations (pp. 94-107, chapter 6). Oxford: University of Oxford. Recuperado em 15 janeiro, 2005, de http://www.sociology.ox.ac.uk/papers/luhmann94-107.doc

Macaulay, S. (1963). Non-contractual relations in business: a primarily study. American Sociology Review, 28(1), 55-67. doi: $10.2307 / 2090458$

MacCurtain, S., Flood, P.C., Ramamoorty, N., West, M., \& Dawson, J. (2009). The top team, trust, reflexivity, knowledge sharing and innovation. Proceedings of the Academy of Management Annual Meeting Proceedings, pp.1-6.

Mayer, R. C.; Davis, J. H., \& Schoorman, F. D. (1995). An integrative model of organizational trust. Academy of Management Review, 20(3), 709-734.

doi: 10.5465/AMR.1995.9508080335

Mesquita, L., \& Lazzarini, S. (2008). Vertical and horizontal relationships in developing economies: implications for SMEs' access to global markets. Academy of Management Journal, 51(2), 359-380. doi: 10.2307/20159513

Osterloh, M., \& Frey, B. S. (2000, September-October). Motivation, knowledge transfer, and organizational forms. Organization Science, 11(5), 538-550.

doi: 10.1287/orsc.11.5.538.15204

Ostrom, E. (2003). Toward a behavioral theory linking trust, reciprocity and reputation. In E. Ostrom \& J. Walker (Eds.), Trust \& reciprocity (pp.19-79). New York: Russell Sage Foundation.
Ouchi, W. G. (1980). Markets bureaucracies and clans. Administrative Science Quarterly, 25(1), 129-141. doi: $10.2307 / 2392231$

Pillai, R. C., Schriesheim, C., \& Williams, E. (1999). Fairness perceptions and trust as mediators for transformational and transactional leadership: a two-study sample. Journal of Management, 25(6), 897-933.

doi: 10.1016/S0149-2063 (99) 00031-8

Ramos, A. G. (1981). A nova ciência das organizações. Rio de Janeiro: Fundação Getulio Vargas.

Reina, D. S., \& Reina, M. L. (2006). Trust and betrayal in the workplace: building effective relationships in your organization (2nd ed.). San Francisco: Berrett Koehler.

Ring, P. S., \& Van De Ven, A. H. (1992). Structuring cooperative relationships between organizations. Strategic Management Journal, 13(7), 483-498.

doi: $10.1002 / s m j .4250130702$

Ripperger, T. (1998). Ökonomik des Vertauens. Tübingen: Mohr Siebeck.

Roberts, J. (2000). From know-how to show-how? Questioning the role of information and communication technologies in knowledge transfer. Technology Analysis and Strategic Management, 12(4), p. 429-443. doi: $10.1080 / 713698499$

Rolland, N., \& Chauvel, D. (2000). Knowledge transfer in strategic alliances. In C. Despres \& D. Chauvel (Eds.), Knowledge horizons: the present and the promise of knowledge management (pp. 225-236). Boston, MA: Butterworth Heinemann.

Sako, M. (1998). Does trust improve business performance? In C. Lane \& R. Bachmann (Eds.), Trust within and between organizations. Oxford: Oxford University Press.

Schoorman, F. D., Mayer, R. C., \& Davis, J. H. (2007). An integrative model of organizational trust: past, present, and future. Academy of Management Review, 32(2), 344-354. doi: 10.5465/AMR.2007.24348410

Ugboro, I. O. (2003, Winter). Influence of managerial trust on survivors' perceptions of job insecurity and organizational commitment in a post restructuring and downsizing environment. Journal of Behavioral and Applied Management, 4(3), 231-253.

Van Den Steen, E. (2003, March). On the origin and evolution of corporate culture. Massachussetts: MIT. Working Paper.

Van De Ven, A. H., \& Walker, G. (1984). The dynamics of interorganizational coordination. Administrative Science Quarterly, 29(4), 598-621. doi: 10.2307/2392941

Whitener, E. M., Brodt, S., Korsgaard, A., \& Werner, J. (1998). Managers as initiators of trust: an exchange relationship framework for understanding managerial trustworthy behavior. Academy of Management Review, 23(3), 513-530. doi: 10.5465/AMR.1998.926624 
Williamson, O. E. (1985). The economic institutions of capitalism - firms, markets, relational contracting. New York: Free Press.

Williamson, O. E. (1995). Organization theory. New York \& Oxford: Oxford University Press.

Williamson, O. E. (1996). The mechanisms of governance. Oxford: Oxford University Press.

Wolff, B. (1996). Constitutional contracting and corporate constitution. In A. Picot \& E. Schlicht (Eds.). Perspectives on contract theory (pp. 95-108). Berlin et al., Springer.

Wolff, B. (2000, Mai). Kalkül und Vertrauen. Zur Institutionalisierung von Moral in der Ökonomik, Manuskript zum Vortrag im Rahmen der Tagung,,Vertrauen in Gesellschaft und Organisationen. Tutzing.

Xavier Molina-Morales, F., Teresa Martínez-Fernandez, M., \& Torló, V. J. (2011). The dark side of trust: the benefits, costs and optimal levels of trust for innovation performance. Long Range Planning, 44(2), 118-133. Recuperado em 14 junho, 2012, (http://www.econbiz.de/Record/the-dark-side-of-trust- the-benefits-costs-and-optimal-levels-of-trust-for-innovationperformance-molina-morales-xavier/10009157074. doi: 10.1016/j.Irp.2011.01.001)

Zanini, M. T. F. (2005, dezembro). Relações de confiança nas empresas da nova economia informacional - uma avaliação dos efeitos da incerteza sobre o comportamento organizacional. Cadernos Ebape.br, 3(4), 1-21.

Zanini, M. T. F. (2007). Trust within organizations of new economy - a cross-industrial study. Wiesbaden, Germany: DUV.

Zanini, M. T. F. (2011). Trust management. In R. Stock-Homburg \& B. Wolff (Eds.), Handbuch Strategisches Personalmanagement (pp. 335-352). Wiesbaden: Gabler Verlag.

Zanini, M. T. F., \& Almeida, A. L. C. (2009). The impact of environmental uncertainty on trust relationships. Revista de Administração da Universidade de São Paulo, 44(4), 313-326.

Zucker, L. G. (1986). Production of trust: institutional sources of economic structure, 1840-1920. Research in Organizational Behavior, 8, 53-111.

\section{The mediating role between trust and organizational performance}

This article seeks to contribute to the discussions about the relationship between trust and organizational performance from an economics perspective. We examine the mediation role between trust and organizational performance by approaching the role of trust as an element of informal coordination within the organizations. We observe that interpersonal trust is central element to better understand relational contracts, like labor contracts, and investments in the creation and maintenance of trust environments should observe an economic rationality. From a literature review, we conclude that trust is an essential element to the execution of specific organizational tasks, and we observe its mediating relationship with organizational performance, trying to better understand the necessity and the benefits of high and low trust social contexts.

Keywords: trust, informal coordination, relational contract, organizational performance.

\section{El papel mediador entre la confianza y el desempeño organizacional}

Este artículo pretende contribuir a los debates sobre el tema de la confianza interpersonal en las organizaciones de una perspectiva económica. Se examinó la relación entre la confianza y el desempeño organizacional a través del análisis del papel de la confianza como un elemento de coordinación informal en la ejecución de diversas tareas de organización. Hemos encontrado que la confianza interpersonal es fundamental para una mejor comprensión de los contratos relacionales, tales como contratos de trabajo, y las inversiones en crear y mantener un ambiente de trabajo basado en la confianza debe cumplir con la racionalidad económica. A través de una revisión de la literatura, se concluye que la confianza es un elemento esencial para la ejecución de determinadas tareas de organización, y observamos su relación con el mediadora con el desempeño, tratando de comprender la necesidad y beneficios de los contextos sociales de alta y baja confianza.

Palabras clave: confianza, coordinación informal, contrato relacional, desempeño organizacional. 